\title{
Polydeoxyribonucleotides Improve Diabetic Wound Healing in Mouse Animal Model for Experimental Validation
}

\author{
Tae-Rin Kwon ${ }^{1}$, Sung Won Han ${ }^{1,2}$, Jong Hwan Kim² ${ }^{2}$ Byung Chul Lee ${ }^{1,2}$, Jae Min Kim ${ }^{1}$ \\ Ji Yeon Hong ${ }^{1}$, Beom Joon Kim ${ }^{1,2}$ \\ ${ }^{1}$ Department of Dermatology, Chung-Ang University College of Medicine, ${ }^{2}$ Department of Medicine, Graduate School, Chung-Ang University, \\ Seoul, Korea
}

Background: Wound healing mechanisms is believed to have effects similar to wound healing disorders in diabetic patients, including abnormal inflammatory cells, angiogenesis disorders, and reduced collagen synthesis. Therefore, reestablishment of structural and promoted angiogenesis could be beneficial to promote wound healing process. Objective: Therefore, we investigated whether the polydeoxyribonucleotide (PDRN) that was self-production in Korea, could be useful as an intradermal injection for promoting wound healing. Also, we validate for wound healing effect of PDRN using healing-impaired ( $\mathrm{db} / \mathrm{db})$ mice. Methods: In this study, we confirmed the effects of PDRN by creating wound models in in vitro and in vivo model. Using an in vitro wound healing assay, we observed that PDRN stimulated closure of wounded monolayers of human fibroblast cells. PDRN $(8.25 \mathrm{mg} / \mathrm{ml})$ or phosphate-buffered saline $(0.9 \% \mathrm{NaCl})$ was injected once daily into the dermis adjacent to the wound for 12 days after skin injury. Results: Time course observations revealed that mice treated with PDRN showed accelerated wound closure and epidermal and dermal regeneration, enhanced angiogenesis. The wound area

Received August 6, 2018, Revised January 2, 2019, Accepted for publication February 12, 2019

Corresponding author: Beom Joon Kim, Department of Dermatology, ChungAng University Hospital, 102 Heukseok-ro, Dongjak-gu, Seoul 06973, Korea. Tel: 82-2-6299-1525, Fax: 82-2-823-1049, E-mail: beomjoon@unitel.co.kr ORCID: https://orcid.org/0000-0003-2320-7621

This is an Open Access article distributed under the terms of the Creative Commons Attribution Non-Commercial License (http://creativecommons. org/licenses/by-nc/4.0) which permits unrestricted non-commercial use, distribution, and reproduction in any medium, provided the original work is properly cited.

Copyright (c) The Korean Dermatological Association and The Korean Society for Investigative Dermatology and depth decreased at 3, 6, 9, and 12 days after skin injury. Histological evaluation showed an increase of vascular endothelial growth factor, CD31, and collagen fibers in the PDRN group compared with the control group, indicating that PDRN was effective in the treatment of delayed wound healing caused by diabetes. Conclusion: This study suggests that our PDRN has a wound healing effect in transgenic animal models with cells and diabetes through angiogenesis.

(Ann Dermatol 31(4) 403 413, 2019)

\section{-Keywords-}

CD31, Diabetes mouse model, Polyribonucleotide, Vascular endothelial growth factor A, Wound healing

\section{INTRODUCTION}

Wound healing is a complex process that is divided into three sequential but overlapping phases of inflammation, proliferation, and remodeling. Migration and growth of epithelial cells, fibroblasts, and vascular endothelial cells are primary components of wound healing ${ }^{1}$. Healing is concomitant with an increased release of angiogenic growth factors from macrophages and keratinocytes, such as vascular endothelial growth factor (VEGF), fibroblast growth factor (FGF), platelet-derived growth factor (PDGF), epidermal growth factor (EGF), and transforming growth factor- $\beta 1$ (TGF- $\beta 1$ ), and impairment of this release leads to a delay in skin repair ${ }^{2}$. Impaired wound healing in diabetics is not completely understood but is believed to include abnormalities in inflammatory cells, impaired neovascularization, decreased synthesis of collagen, and increased levels of proteinases ${ }^{2,3}$. In particular, a chronic 
wound tends to show decreased levels of EGF, FGF, TGF- $\beta$, PDGF, and VEGF, interleukin (IL)- 1 and -6 , and tumor necrosis factor- $\alpha$ (TNF- $\alpha)^{4}$. Various drugs have been proposed to address these problems. Currently being developed or studied as injectable wound healing agents are adipocyte derived interstitial cells ${ }^{5}$, growth factors such as EGF, $\mathrm{PDGF}^{6,7}$, and placenta injections ${ }^{8,9}$.

Polydeoxyribonucleotide (PDRN), which is produced by a high temperature extraction process from the proteins of trout sperm, is a mixture of purines, pyrimidines, deoxyribonucleotides, and deoxyribonucleosides and has varying lengths of 50 to 2,000 base pairs. It consists of low molecular weight DNA with no more than $95 \%$ protein $^{10-12}$.

PDRN has shown remarkable therapeutic efficacy by promoting angiogenesis through increasing VEGF expression during skin flap techniques, burn wound healing, and recovery of diabetic wound delays ${ }^{2,10,13,14}$. Furthermore, the effects of PDRN were investigated in a model of diabetesimpaired wound healing ${ }^{2}$. PDRN improved the skin repair process and enhanced wound breakage strength in diabetic animals. Another clinical situation characterized by a poor skin repair process and impaired angiogenesis is thermal injury ${ }^{13,15}$. The effects of PDRN were investigated in mice with deep-dermal, second degree burn injuries, and the treatment enhanced burn wound re-epithelialization and decreased time to final wound closure ${ }^{2,3}$.

PDRN has been shown to activate A2A receptor ${ }^{16}$. PDRN exerts anti-inflammatory effects by inhibiting mast cell degranulation and inflammatory cytokines. A previous study reported that PDRN administration reduced pro-inflammatory mediators, such as TNF- $\alpha$, IL-6, and High-mobility group protein $1^{3}$. PDRN markedly increased upstream signaling for VEGF, as well as the mature protein in wounds, indicating its ability to improve the healing of wounds in genetically diabetic mice that exhibit wound healing impairment due to defects in VEGF regulation at the gene expression level $^{2,17,18}$.

In this study, we used PDRN extracted (providing BR Beautiful Revolution Co., Ltd., Wonju, Korea) from the testis of Oncorhynchus masou and was produced in Korea by itself. Currently, existing PDRNs are extracted from the semen of O. masou or testis of Oncorhynchus keta. In previous experiments, investigators injected PDRN into the abdominal cavity, but we established experimental differences by injected intra-dermally around the wound at injection sites. Therefore, this study was conducted to test whether the PDRN used in our experiment is usable as an injectable agent and focused on exploring the hypothesis that PDRN is effective in treating the delayed wound healing of diabetic mice by increasing the expression of VEGF and CD31.

\section{MATERIALS AND METHODS}

\section{Cell culture}

We purchased Normal Human Primary Dermal FibroblastsNeonatal (HDF-n), isolated from human neonatal foreskin, from GIBCO $^{\circledR}$ (Invitrogen, Carlsbad, CA, USA). We injected human fibroblasts into the bottom of cell-culture dishes and added Dulbecco's Modified Eagle's Medium (DMEM; Welgene, Gyeongsan, Korea) containing penicillin (100 $\mathrm{IU} / \mathrm{ml})$, streptomycin $(100 \mu \mathrm{g} / \mathrm{ml})$, and $10 \%$ fetal bovine serum $\left(\mathrm{GIBCO}^{\mathbb{R}}\right)$. Then, we cultivated the dishes in an incubator with a $5 \% \mathrm{CO}_{2}$ atmosphere at $37^{\circ} \mathrm{C}$.

\section{Cell viability determination}

HDF-n cells $\left(5 \times 10^{3}\right.$ cells $)$ were seeded into a microtiter plate with 96 wells and cultivated for 24 hours under standard cell culture conditions. We removed the media and rinsed the plates with Dulbecco's phosphate-buffered saline (DPBS). We then induced starvation and maintained it for 24 hours. Next, we added new media with PDRN (except for media supplements) into the plate and cultivated the cells for 24 hours. After this, we again removed media with PDRN and rinsed the plate with DPBS. To assess cell viability, we diluted the CCK-8 reaction solution with media with no added supplements at a ratio of $1: 10$, added $100 \mu \mathrm{l}$ of the diluted solution into each well, incubated the cells for one hour, and measured absorbance at $450 \mathrm{~nm}$. To assess cell proliferation, we measured cell viability at 24,48 , and 72 hours.

\section{Assessment of cell migration}

We seeded HDF-n cells in Ibidi $^{\text {TM }}$ chambers in $60 \mathrm{~mm}$ dishes at a density of $1.0 \times 10^{4}$ cells/chamber and cultivated them for 24 hours under standard cell culture conditions. Then, we removed the chambers, rinsed them with DPBS, each plate added 25, 50, 100, and $200 \mu \mathrm{g} / \mathrm{ml}$ of PDRN with the media, and cultivated them under standard cell culture conditions. We observed cell migration with an optical microscope at each time point $(0,24,48$, and 72 hours).

\section{Animals}

Every animal experiment conducted for this study was approved by Chung-Ang University's Institutional Animal Care and Use Committee (approval number: 2014-00007). We purchased seven-week-old BKS.Cg- $+L e p r^{d b} /+L e p r^{d b}$ and $^{m}+/+$ Lepr $^{d b}$ female mice strains from The Jackson Laboratory in Korea. Diabetic mutations are the result of spontaneous point mutations in the leptin receptor gene 
(Lepr). This mouse becomes obese after 3 to 4 weeks. The rise in plasma insulin begins at 10 to 14 days based on the information provided by the vendor, and blood glucose begins at 4 to 8 weeks. All mice were housed in personally air-conditioned animal rooms with standard environmental controls of $24^{\circ} \mathrm{C} \pm 2^{\circ} \mathrm{C}$ (temperature), $50 \% \pm 10 \%$ (humidity), and a 12-hour day/night cycle. Animals were allowed free access to food and underwent a one-week stabilization period before testing.

\section{Wound model}

Mice were anesthetized with Zoletil (30 mg/kg) and Rompun (10 mg/kg). The back of each mouse was divided into two areas according to the centerline along the back. Each area was inflicted with a full-thickness skin wound 4 $\mathrm{mm}$ in diameter using a biopsy punch (Kai Industries Co., Ltd., Tokyo, Japan). The size of a wound area was measured right after the wound was inflicted by a 4-mm biopsy punch, in consideration of skin contraction. The size of the wounds inflicted by a 4-mm biopsy punch was 12.56 $\mathrm{mm}^{2}$, which is within one standard deviation of the average size of the wounds of all mice $\left(12.83 \pm 0.83 \mathrm{~mm}^{2}\right)$. As a result, each mouse had two wounds, one serving as the control and the other as the test. The PBS and PDRN group also had their wounds injected with $50 \mu$ I into the wound edges of each of the wound sites on each animal in groups of two, using a $0.5 \mathrm{ml}$ syringe and a $28.5 \mathrm{G}$ needle. Each wound was dressed, and PDRN were applied once a day. We observed mice for general symptoms once a day until the test was complete. Observations were made six hours after PDRN intradermal injection. To minimize contamination of the wounds and prevent the PDRN from migrating and dropping out, we used Tegaderm ${ }^{\mathrm{TM}}$ films (3M, St. Paul, MN, USA). When $90 \%$ of more of the wound was regenerated, the test was considered complete, and the mice were humanely euthanized.

\section{Measurement of the size and depth of wounds}

We observed the surface change in the wounds of every mouse using a DSLR (D3200; Nikon, Tokyo, Japan) and Folliscope (LeedM, Seoul, Korea) at days 0, 3, 6, 9, and 12. We captured three-dimensional (3D) images of the wounds with a PRIMOS ${ }^{\text {LITE }}$ optical 3D skin measuring device (LMI Technologies GmbH, Teltow, Germany) and analyzed them with PRIMOS software version 5.7. We measured the size and depth of the wounds to assess the time required for wound healing.

\section{Histological assessment}

After the mice were euthanized, we extracted skin tissue from the wounds and fixed them with 10\% neutral-buf- fered formalin fixative. We embedded the tissues in molten paraffin wax and the embedded tissues were sectioned into $5-\mu \mathrm{m}$ slices. We stained the slices with hematoxylin and eosin $(\mathrm{H} \& \mathrm{E})$ to observe infiltration of inflammatory cells, location of the muscular layer, numbers of foreign body reactions and inflammatory cells, re-epithelialization, proliferation of fibroblasts, and formation of blood vessels. We used Masson's trichrome and van Gieson's staining protocols to determine whether collagen and elastic fibers were created at the wound site.

\section{Immunohistochemistry analysis}

VEGF and CD31 immunostaining was performed using tissue samples collected from day 12 after the surgical procedure in order to investigate angiogenesis. Using immunohistochemical staining for VEGF and CD31, the slides from each group were assessed for intensity of expressed diaminobenzidine (DAB) chromogen. CD31 represents a highly specific marker for vascular and endothelial cells.

Paraffin-embedded tissues were sectioned (5 $\mu \mathrm{m})$, and the slides were subjected to antigen retrieval, with endogenous peroxidase activity quenched using hydrogen peroxide. After blocking non-specific proteins with normal serum in PBS (0.1\% Tween-20), slides were incubated with rabbit polyclonal antibodies against CD31 (ab28364; Abcam, Cambridge, UK) or VEGF (SC-152; Santa Cruz Biotechnology, Santa Cruz, CA) (both at a 1:50 dilution). After washing with PBS, slides were incubated with the appropriate biotinylated secondary antibody, followed by conjugated horseradish peroxidase-labeled streptavidin (Thermo Fisher, Waltham, MA, USA), and then washed with PBS. The slides were then incubated with $\mathrm{DAB}$ as the chromogen, followed by counterstaining with diluted Harris' H\&E and mounting with coverslips. After staining, three high-power fields $(\times 400)$ were randomly selected in each slide, and the average proportion of positive cells in each field was counted using the true color multi-functional cell image analysis management system (Image-Pro plus 7.0; Media Cybernetics Inc., Silver Spring, MD, USA). To rule out any non-specific staining, PBS was used instead of the primary antibody as a negative control.

\section{Statistical analysis}

Data are expressed as the mean plus or minus the standard error of the mean (mean \pm SEM), and statistical tables in this document contain figures as well as the mean of each group for each statistical period. Comparisons between the control group and the test group were carried out using PASW Statistics ver. 18.0 (IBM Corp., Armonk, NY, USA). The Shapiro-Wilk test was used to test whether 
normal distributions were present in the data. Probability errors were measured by the Kruskal-Wallis one-way analysis of variance (ANOVA) on ranks. In all cases, a probability error $<0.05$ was selected as the criterion for statistical significance. When comparisons with the control group were made, the Mann-Whitney $U$ test and Student's t-test using independent samples were used. Probability errors are expressed as follows: $p<0.05, p<0.01, p<$ 0.001 .

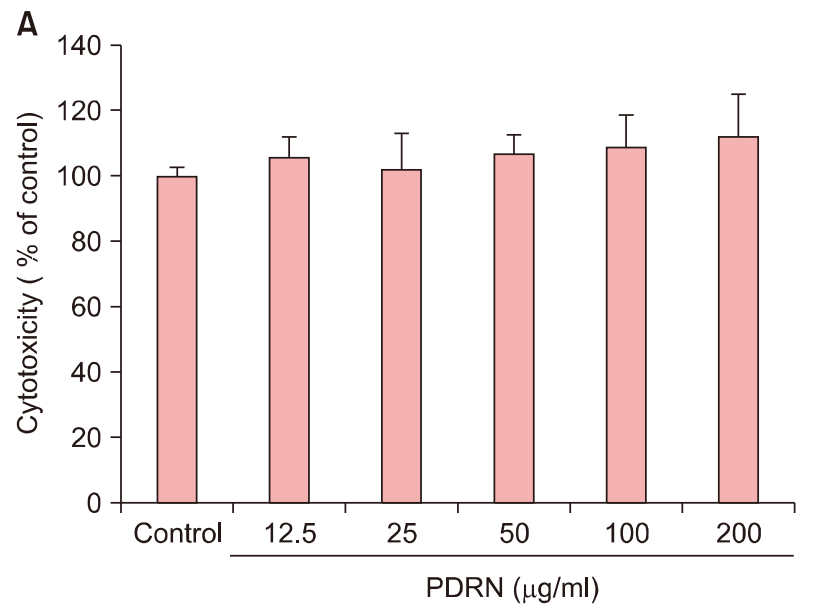

\section{RESULTS}

PDRN has no cytotoxic effects and enhances proliferation in human dermal fibroblast neonatal cells

We measured the cytotoxicity of the PDRN using CCK-8 reaction solution, which can determine the viability of cells, in human dermal fibroblast neonatal (HDF-n) cells. The percentage of viable cells was expressed as a proportion of control cell viability, which was set to $100 \%$. The control group was comprised of untreated HDF-n

B

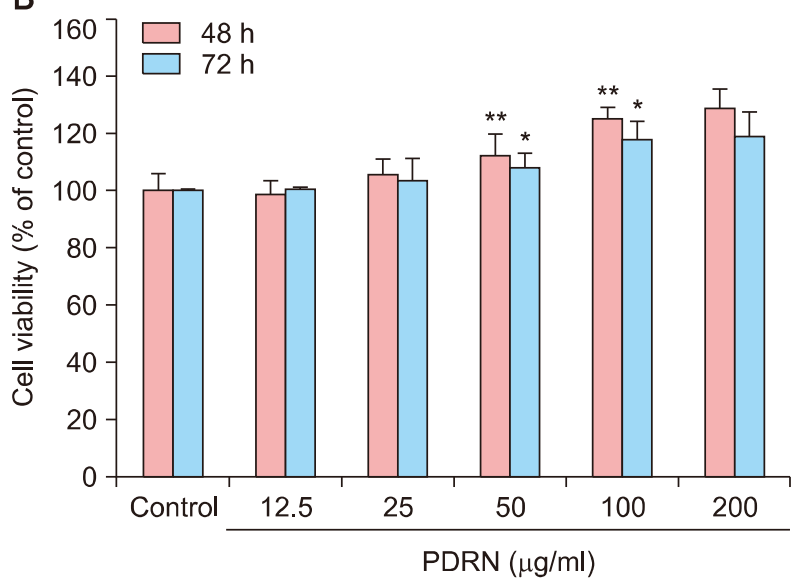

C

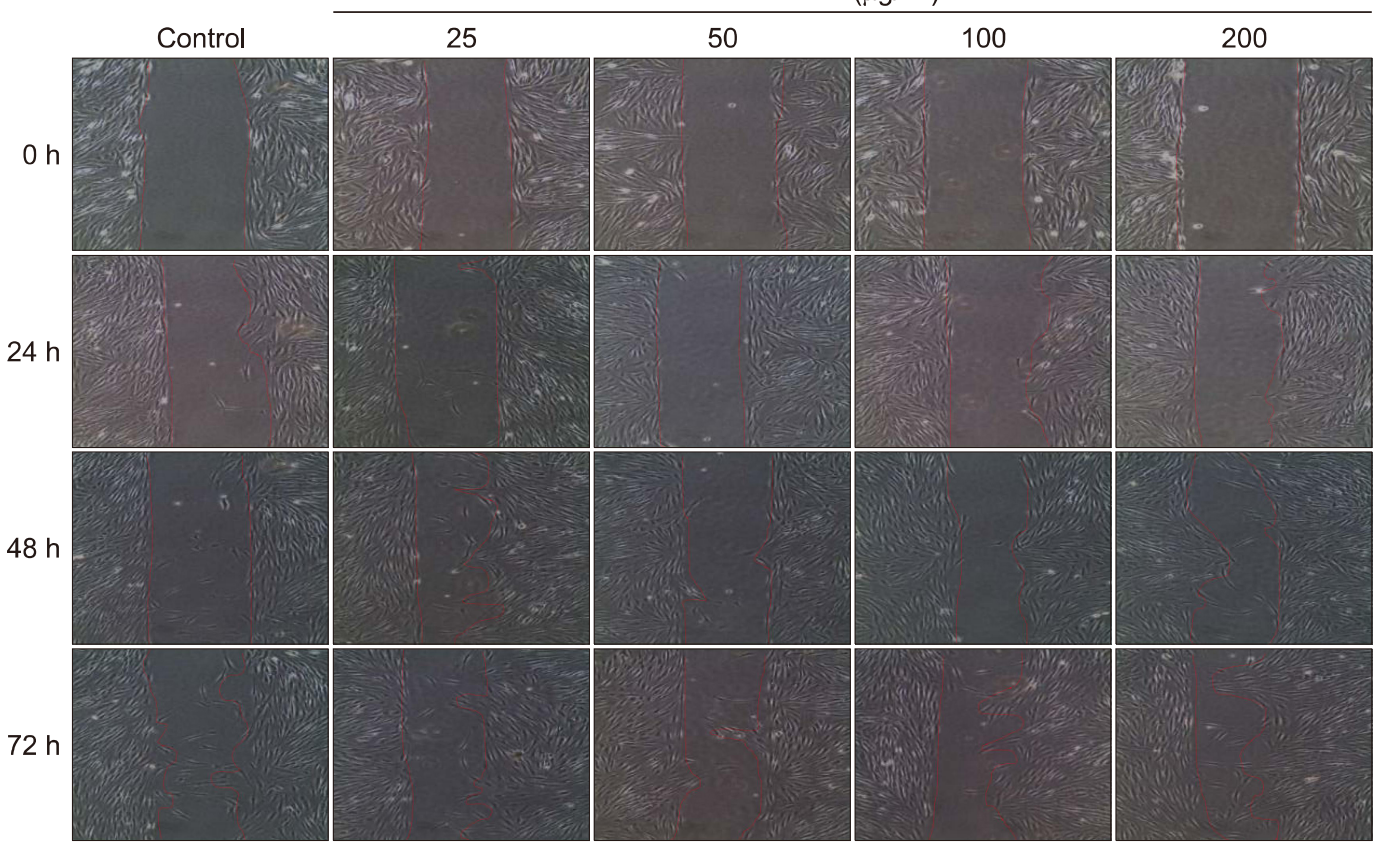

Fig. 1. Effects of polydeoxyribonucleotide (PDRN) on cytotoxicity and proliferation in human dermal fibroblast neonatal (HDF-n) cells. (A) The cytotoxicity of PDRN was measured using CCK-8 reaction solution in on HDF-n cells. The percentage of viable cells was expressed as a proportion of control cell viability, which was set to $100 \%$. (B) The cell proliferation of HDF-n cells at 48 and 72 hours. The number of HDF-n cells treated with PDRN. (C) The cell migration of HDF-n. We observed these results with optical microscopes (CKX41; OLYMPUS, Tokyo, Japan) at each time point $(0,24,48$, and 72 hours). Significant differences from the control group are ${ }^{*} p<0.05,{ }^{* *} p<0.01$. 
cells, while the other groups consisted of HDF-n cells treated with PDRN at a concentration of 12.5, 25, 50, 100 , or $200 \mu \mathrm{g} / \mathrm{ml}$. To assay cell proliferation in the cells treated with PDRN, we first determined the concentration of PDRN at which no cytotoxicity was observed and then measured cell proliferation in cells treated with that concentration after 48 and 72 hours. When treated with PDRN at concentrations of $12.5,25,50,100$, and $200 \mu$ $\mathrm{g} / \mathrm{ml}$, the number of HDF-n cells increased by $98.4 \%$, $105.4 \%, 112.6 \%, 125.2 \%$, and $130.1 \%$, respectively, 48 hours after treatment and by $100.5 \%, 103.7 \%, 107.8 \%$, $117.8 \%$, and $120.4 \% 72$ hours after treatment (Fig. 1). We observed migration of HDF-n cells using an optical microscope at each time point (0, 24, 48, and 72 hours). Compared with control cells, the space between sides of the scratched wound became narrower after 48 hours in cells treated with PDRN, but this difference was not statistically significant. Together, these results show that PDRN has no cytotoxic effect and enhances cell proliferation.

\section{PDRN improves wound healing in diabetic mice}

We conducted a visual inspection to assess the progress of wound closure. There was no significant difference be- tween the PBS-injected group and the PDRN-injected group in the ${ }^{m}+/+$ Lepr $^{d b}$ mice (hetero type) however + Lepr $^{d b} /$ + Lepr $^{\mathrm{db}}$ mice $(\mathrm{db} / \mathrm{db})$ showed significant increase in granulation tissue and capillary blood vessels in the PDRN injected group compared to PBS injected group (Fig. 2). At the same time as the visual inspection, we also measured and analyzed the size and depth of wounds and the associated decreases (Fig. 3). As for the ${ }^{m}+/+L e p r^{d b}$ mice, those injected with PBS and those with PDRN showed no significant differences in terms of the size of wound areas and wound depth. However, for $+L e p r^{d b} /+L e p r^{d b}$ mice, those injected with PDRN showed a significant decrease in wound depth compared with those injected with PBS at day $6(40.33 \% \pm 3.61 \%, p<0.05)$ and day $9(22.75 \% \pm$ $1.95 \%, p<0.05)$.

\section{PDRN promotes wound healing with rapid re-epitheli- zation}

H\&E revealed that the wounds were recovered in order of PDRN injected group, PBS injected group in the ${ }^{m}+1$ + Lepr $^{d b}$ mice and PDRN injected group, PBS injected group in the $+L e p r^{d b} /+L e p r^{d b}$ mice in the order of date (Fig. 4). In the ${ }^{m}+/+$ Lepr $^{d b}$ mice group, the difference between PBS injected group and PDRN injected group was
A

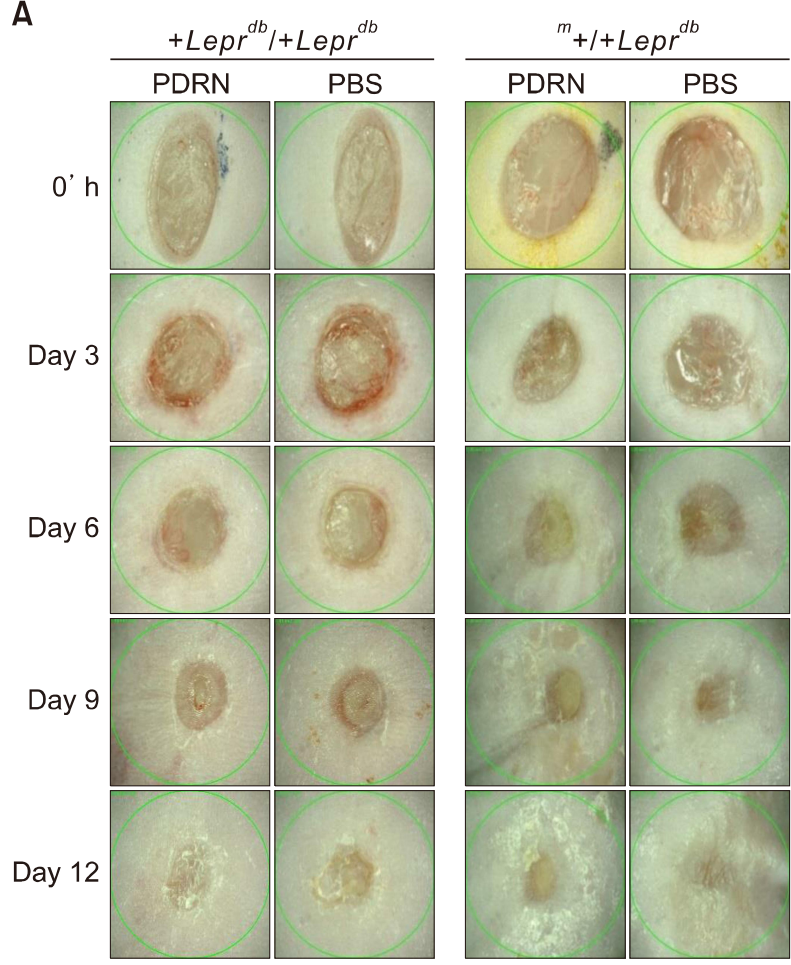

B

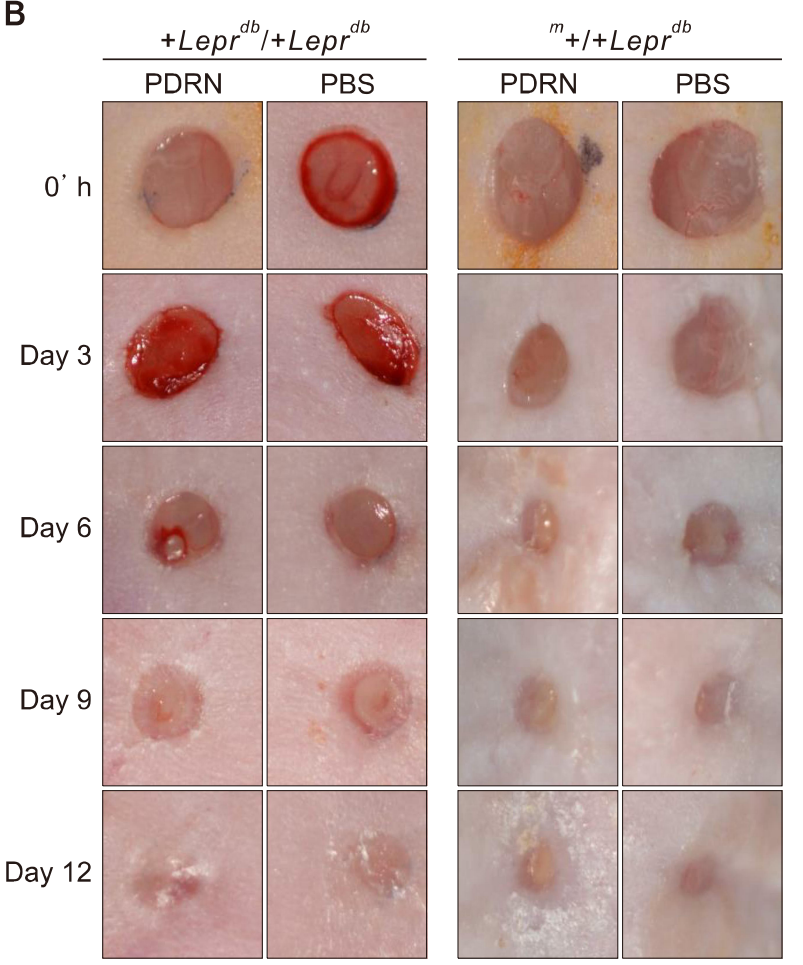

Fig. 2. Polydeoxyribonucleotide $(P D R N)$ stimulates wound healing at diabetic mouse model. (A) Folliscope images $(\times 15)($ LeedM, Seoul, Korea). (B) Digital camera images (D3200; Nikon, Tokyo, Japan). It was confirmed that the area of the wounds was reduced visually with time after skin damage. PBS: phosphate-buffered saline. 
A
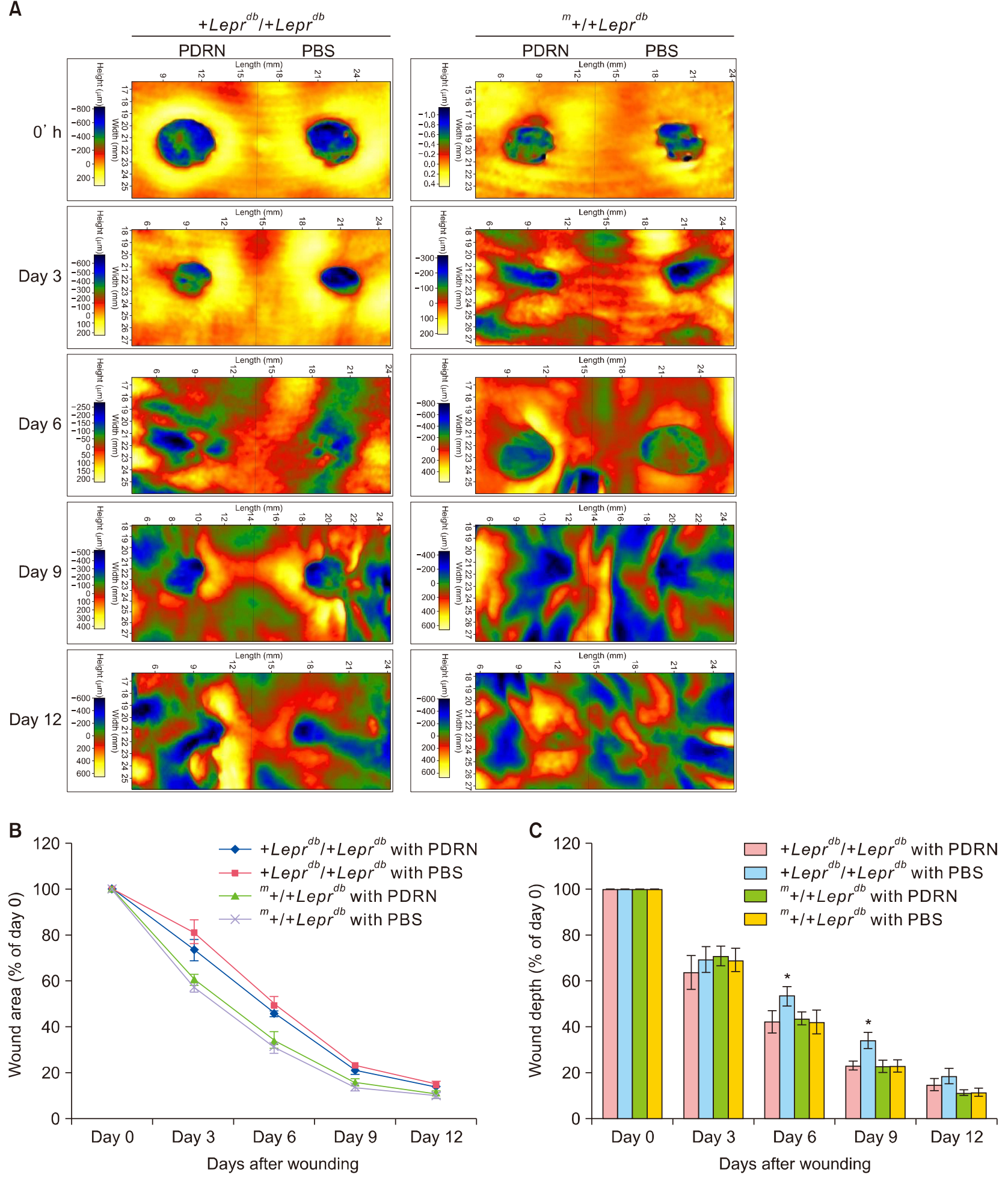

Fig. 3. Polydeoxyribonucleotide (PDRN) decreases wound area and wound depth in diabetic mice. (A) Color-coded surface topography image captured by a PRIMOS ${ }^{\text {LIT }}$ optical 3D skin measuring device, (B) graph showing changes in wound area over time after injury. In $+L e r^{d b} /+L e p r^{d b}$ mice, the reduction of the wound area in the PDRN-injected group occurred more rapidly than in the phosphatebuffered saline (PBS)-injected group. In both ${ }^{m}+/+L e p r^{d b}$ mice and $+L e p r^{d b} /+L e p r^{d b}$ mice, the wound area decreased faster than in controls, but this difference was not significant. (C) Graph showing changes in wound depth over time after injury. The PDRN-injected + Lepr $^{d b} /+$ Lepr $^{d b}$ mice recovered at a rate similar to that of PDRN-injected ${ }^{m}+/+$ Lepr $^{d b}$ mice, but the recovery of the wound depth was delayed in the PBS-injected $+L e p r^{d b} /+L e p r^{d b}$ mice. The wound depth of the PBS-injected group was significantly different from that of the PDRN-injected group on days 6 and $9\left({ }^{*} p<0.05\right)$ in $+L e p r^{d b} /+L e p r^{d b}$ mice. 


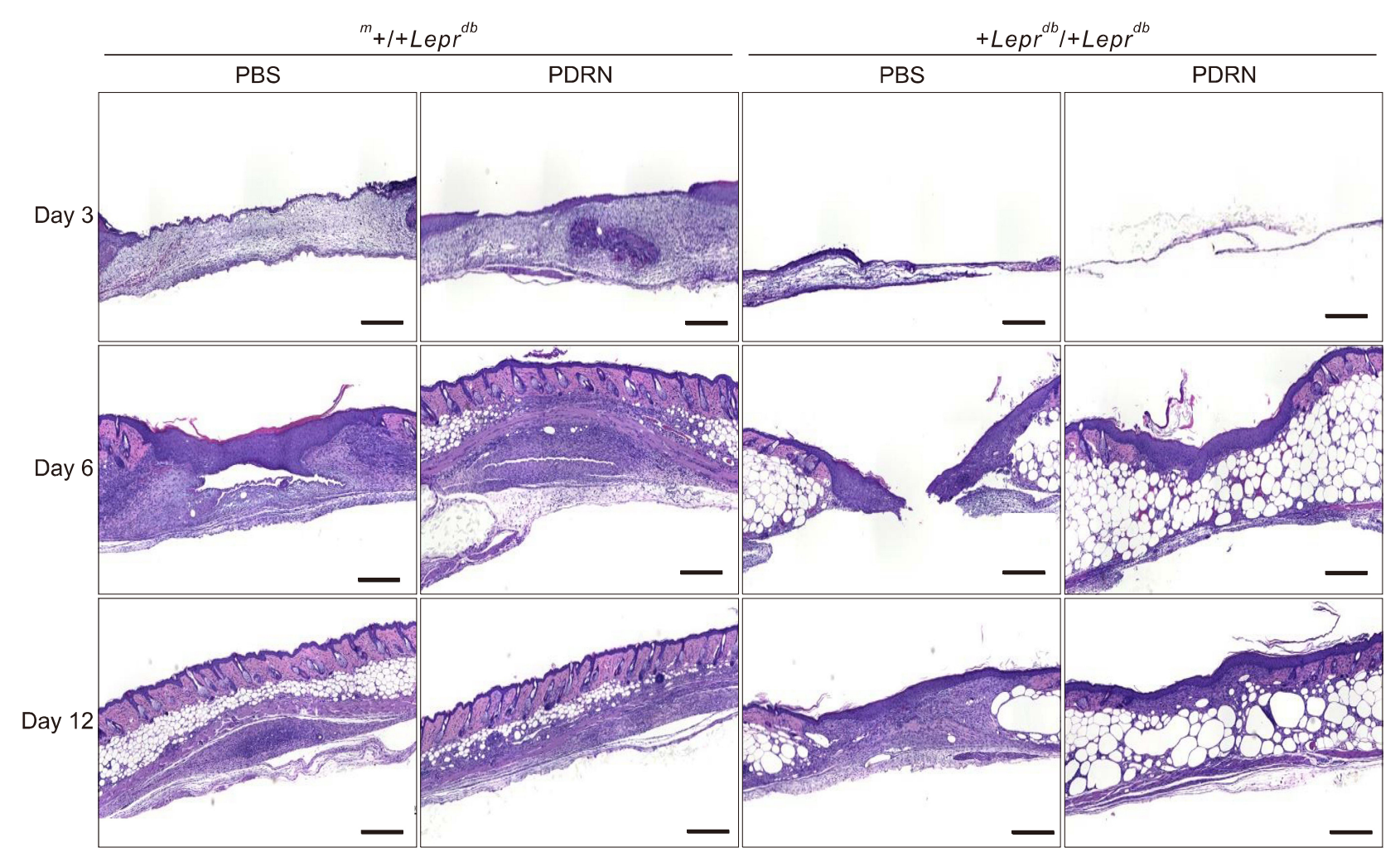

Fig. 4. Polydeoxyribonucleotide (PDRN) promotes wound healing with rapid re-epithelization. Hematoxylin-eosin (H\&E) staining was performed on paraffin block sections of the skin tissue 3, 6, 12 days after initial wounding. H\&E staining revealed that the wounds were recovered in order of PDRN injected group, phosphate-buffered saline (PBS) injected group in the ${ }^{m}+/+$ Lepr $r^{d b}$ mice and PDRN injected group, PBS injected group in the $+\operatorname{Lepr}^{d b} /+L e p r^{d b}$ mice in the order of date. In the ${ }^{m}+/+$ Lepr $^{d b}$ mice group, the difference between PBS injected group and PDRN injected group was not significant in visual examination, but tissue evaluation showed a clear difference between PBS injected group and PDRN injected group. Scale bars $=1 \mathrm{~mm}$.

not significant in visual examination, but tissue evaluation showed a clear difference between PBS injected group and PDRN injected group. The proliferation of fibroblasts was promoted at the wound site, the epidermis with re-epithelialization thickened, and the wound quickly closed. Day 12 after wound, hair follicles were formed normally, and skin thickness was restored normally in the ${ }^{m}+$ l $+\operatorname{Lepr}^{d b}$ mice. Thus, in the $+\operatorname{Lepr}^{d b} /+\operatorname{Lepr}^{d b}$ mice, the proliferation of fibroblasts and keratinocytes in the PDRN-injected group compared to the PBS-injected group was confirmed to be rapidly restored.

\section{PDRN helps to increases collagen synthesis}

In the hetero-type ${ }^{m}+/+$ Lepr $^{d b}$ mice, those injected with PBS and those with PDRN showed no significant histological differences (Fig. 5). However, for diabetic + Lepr $^{d b} /$ + Lepr $^{d b}$ mice, those injected with PDRN showed epithelialization of the epidermis around the wound. In addition, since collagen fibers (the main component of the extracellular matrix) grew relatively quickly and new capillary networks were formed and expanded, tissues at the wound site rapidly achieved a normal morphology.

PDRN promotes therapeutic angiogenesis by increased expression of VEGF and CD31 in a diabetic mouse model

When the intensity of DAB in the VEGF antibody was analyzed, the hetero-type ${ }^{m}+1+\operatorname{Lepr}^{d b}$ mice, those PBS injected group was $38.52 \pm 0.60 \%$, PDRN injected group was $67.21 \% \pm 4.21 \%$, and diabatic $+\operatorname{Lepr}^{d b} /+\operatorname{Lepr}^{d b}$ mice, those PBS injected group was $24.38 \% \pm 1.55 \%$, PDRN injected group was $32.57 \% \pm 1.40 \%$. Both hetero-type and homozygous types mice showed a significant increase $(p<$ 0.01) in PDRN injected group compared to PBS injected group. Also, when the intensity of DAB in the CD31 antibody was analyzed, the hetero-type ${ }^{m}+1+\operatorname{Lepr}^{d b}$ mice, those PBS injected group was $47.36 \% \pm 0.68 \%$, PDRN injected group was $58.051 \% \pm 2.68 \%$, and diabatic $+L e p r^{d b}$ / $+\operatorname{Lepr}^{d b}$ mice, those PBS injected group was $8.98 \% \pm$ $1.60 \%$, PDRN injected group was $13.96 \% \pm 0.64 \%$. Both hetero-type and homozygous types mice showed a significant increase $(p<0.01)$ in PDRN injected group compared to PBS injected group (Fig. 6). 
A
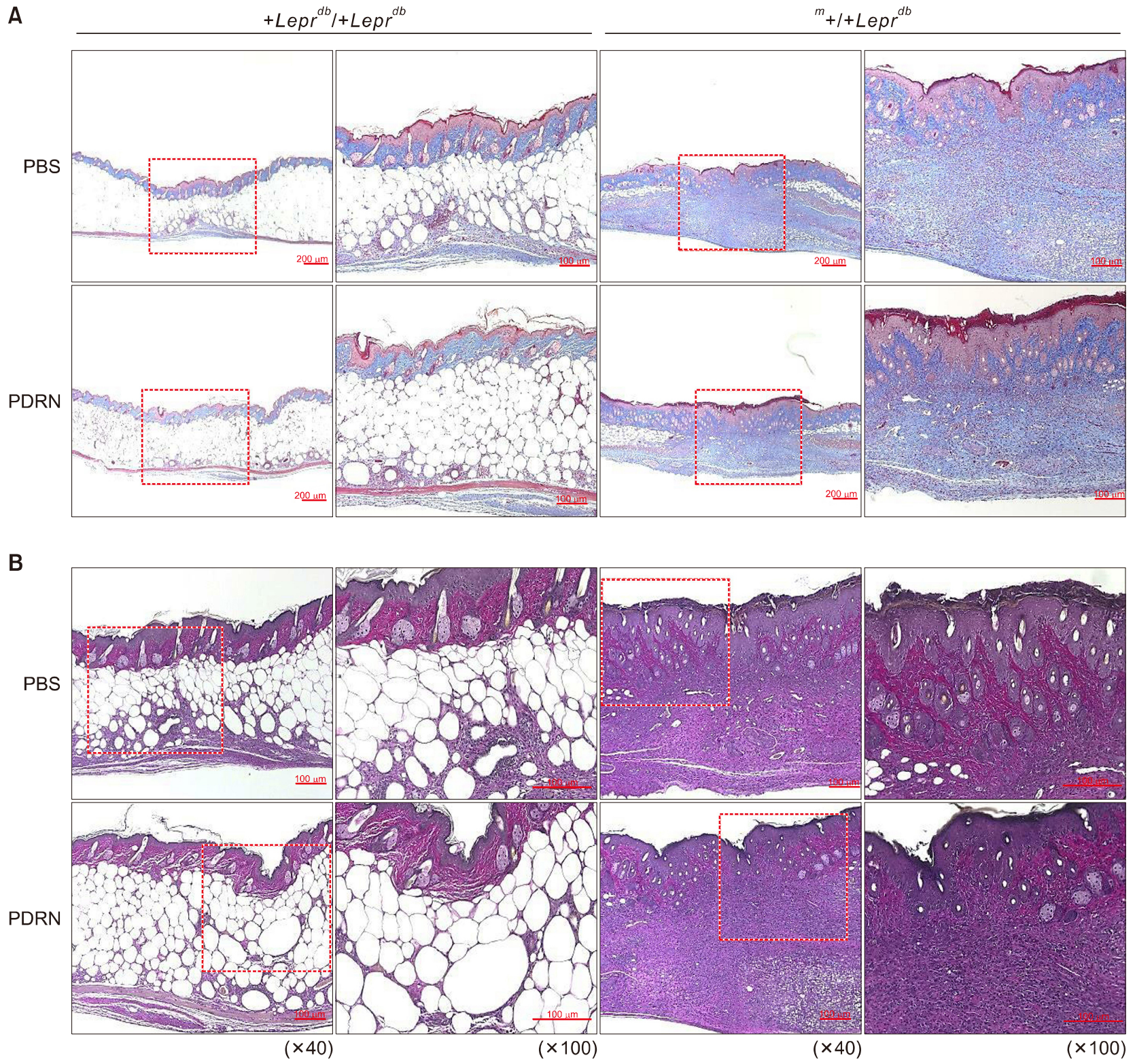

Fig. 5. Polydeoxyribonucleotide (PDRN) promotes skin repair in diabetic mice. (A) Masson's trichrome stain. Red: keratin \& muscle fiber, blue: collagen \& bone, pink: cytoplasm $(\times 40)$. (B) van Gieson's stain. Blue: nuclei, bright red: collagen, yellow: cytoplasm, muscle, fibrin, and red blood cells. For the hetero-type ${ }^{m}+/+\operatorname{Lepr}^{d b}$ mice, those injected with phosphate-buffered saline (PBS) and those with PDRN showed no significant differences histologically. However, for diabetic BKS.Cg- $+L e p r^{d b} /+L e p r^{d b}$ mice, those injected with PDRN showed faster epithelialization of the epidermis around the wound and faster formation of collagen fiber grows compared with those injected with PBS $(\times 100)$.

\section{DISCUSSION}

Common wound healing processes consist of complex interactions among many factors that work together to restore damaged skin. However, in a basic disease state such as diabetes, normal wound healing does not occur, which can lead to chronic wounds ${ }^{19}$. In particular, diabetic foot ulcers affect $15 \%$ of diabetic patients, with most of these cases requiring amputation ${ }^{20,21}$. Therefore, de- layed wound healing in diabetic patients is a threatening and important challenge. Research on treatments for chronic delayed wound healing, such as diabetic wound healing, is ongoing. There is evidence that adequate blood glucose control in diabetic patients can have a significant impact on the rate of wounding ${ }^{22}$. Especially, PDGF-BB has already been approved by the FDA and is currently used in the treatment of chronic ulcers ${ }^{4}$. Living cell therapy can be considered as simultaneous multiple growth 
A

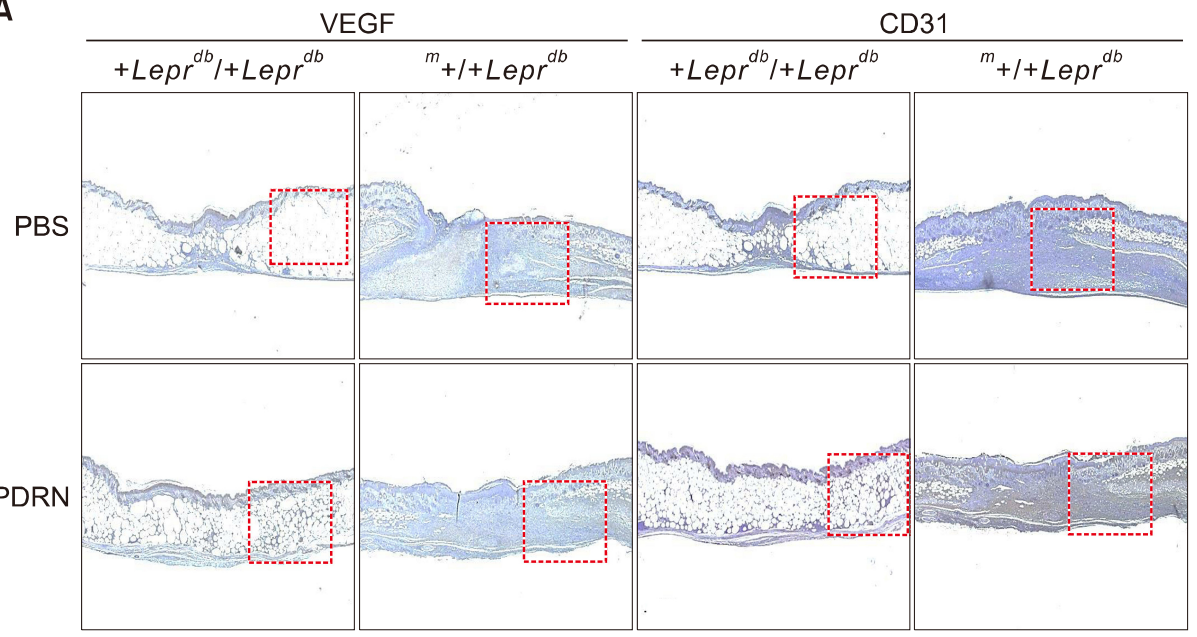

B

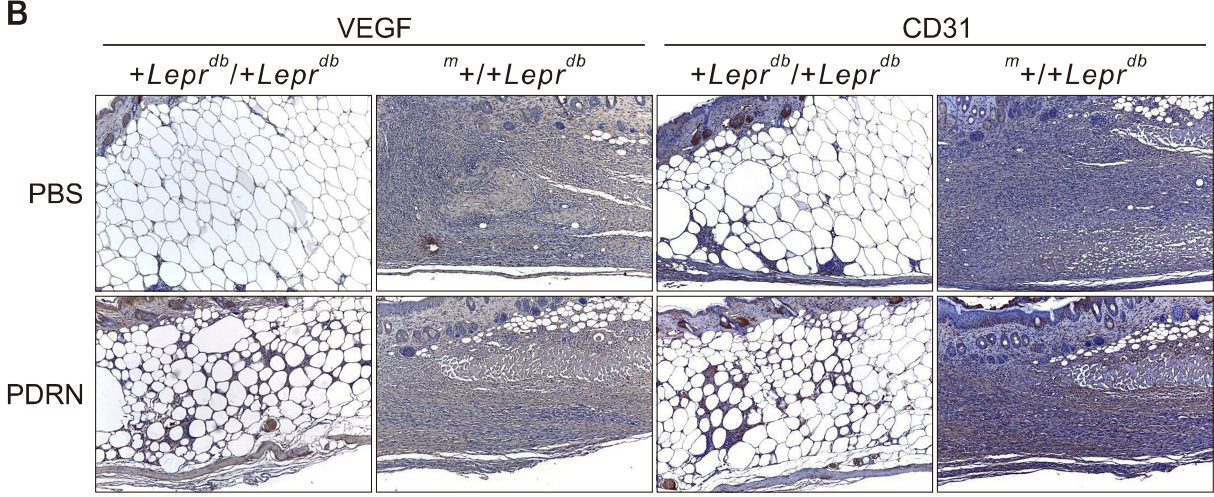

C

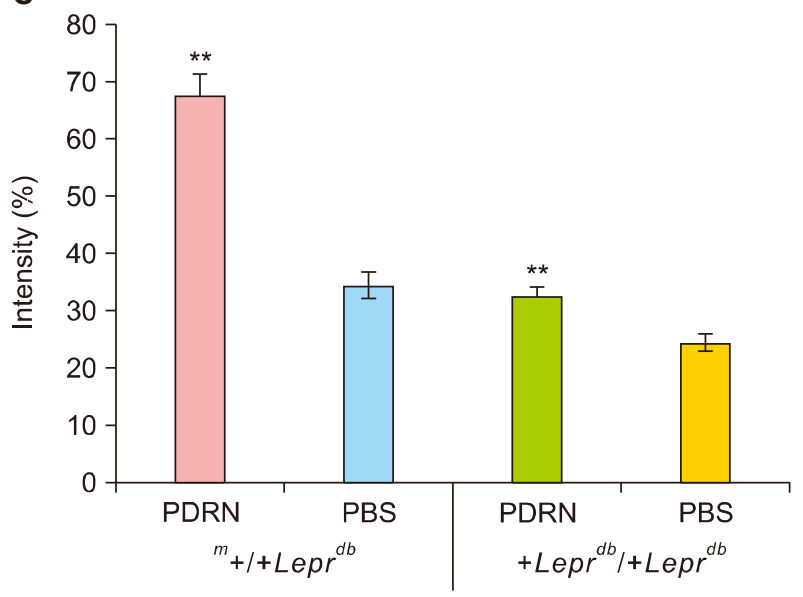

D

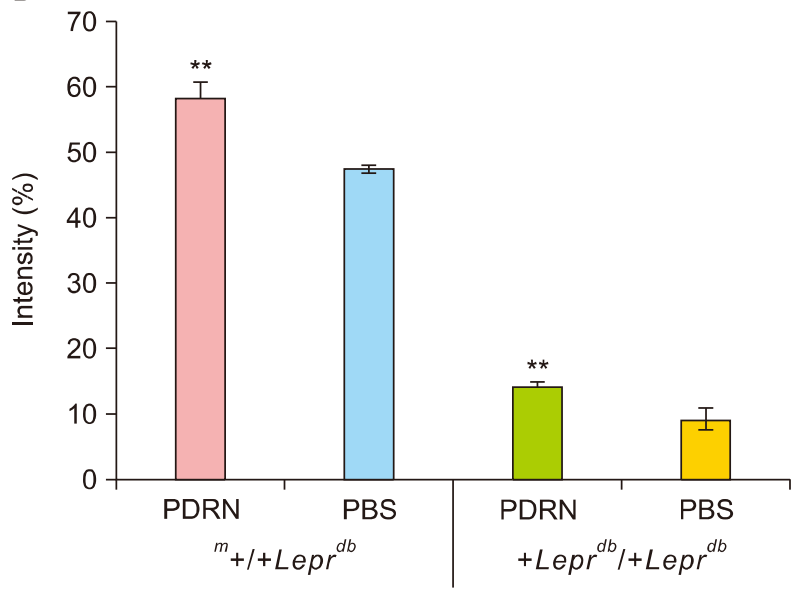

Fig. 6. Polydeoxyribonucleotide (PDRN) increases expression of vascular endothelial growth factor (VEGF) and CD31 in the regenerating wounds of diabetic mice. Expression levels of VEGF and CD31 in the tissues of animals sacrificed at day 12 after skin injury were confirmed by immunohistochemical staining. (A) VEGF and (B) CD31. (C) Graph of VEGF intensity. (D) Graph of CD31 intensity. In both ${ }^{m}+/+L e p r^{d b}$ mice and $+L e p r^{d b} /+L e p r^{d b}$ mice, the PDRN treatment group showed significantly higher expression of VEGF and CD31 than the PBS treatment group (analysis by Image-Pro plus 7.0), one-way ANOVA (Shapiro-Wilk $p>0.05$ ), independent t-test assay $(* * p<0.01$ ).

factor therapy. Nonetheless, there are many reports of administering particular growth factors, cytokines, proteins, or hormones and achieving remarkable results in various models of wound healing, including all of the growth factors and further targets described above ${ }^{23,24}$.

PDRN can stimulate VEGF production under the patho- 
logical conditions of low tissue perfusion and acts through stimulation of the A2A receptor. In addition, acute and chronic toxicity studies have shown a good safety profile. PDRN has been shown to be effective in experimental models of peripheral arterial occlusive disease, damaged wound healing, and thermal injury ${ }^{2,10,13,25,26}$. Based on this information, our study attempted to determine the effects of direct injection of PDRN on the healing of diabetic wound.

We confirmed that, when HDF-n cells were treated with $200 \mu \mathrm{g} / \mathrm{ml}$ of PDRN, there was no cytotoxicity, and cell proliferation increased in a dose-dependent manner at 48 and 72 hourrs. Then, we evaluated the proliferative capacity of dermal fibroblasts, then, we evaluated the proliferative capacity of dermal fibroblasts, we confirmed the effect of PDRN concentration at $50 \mu \mathrm{g} / \mathrm{ml}, 100 \mu \mathrm{g} / \mathrm{ml}$ on fibroblast proliferation, and we verified that dermal fibroblast migration was increased compared to the control group.

Therefore, we hypothesized that PDRN treatment would increase the proliferation of dermal fibroblasts, which would lead to an increase in intra-epidermal collagen fibers. For this test, heterozygote ${ }^{m}+/+$ Lepr $^{d b}$ mice and homozygous $+\operatorname{Lepr}^{d b} /+\operatorname{Lepr}^{d b}$ mice were selected to eliminate the possibility that wound healing of wild-type normal mice through scar contraction would affect the test. And we investigated the mechanisms of this process by establishing a wound healing model in a diabetic mouse model.

As a result, we found that PDRN can improve wound healing in diabetic mice; wound depth was significantly reduced in the PDRN-injected group compared to the PBS-injected group. In the visual evaluation, the difference between the wound healing groups was not significant. However, H\&E staining showed that the wound healing of the PDRN injected group was faster than that of the PBS injected group in both heterozygous and homo mice. Masson's trichrome staining and van Gieson staining revealed that the epidermal layer of the wound was completely regenerated, and that the rapid growth of collagen fibers, the main constituent of the extracellular matrix, prompted the maturation of the wound tissue to normal tissues in PDRN-injected mice.

Also, immunohistochemical stain results suggested that angiogenesis was promoted in the diabetic mouse PDRNinjected group through an increase in expression of VEGF and CD31 compared to the control group. Although various growth factors are involved in the wound healing process, adequate amounts of growth factors must be secreted in a timely manner for proper wound healing to occur. Therefore, we examined VEGF and CD31 expression at the latest time point (day 12) in our study. There- fore, in the $+L e p r^{d b} /+L e p r^{d b}$ mice, PDRN is expected to induce sustained and enhanced expression of VEGF through stimulation of the $\mathrm{A} 2 \mathrm{~A}$ receptor, and it can be argued that the increased expression of VEGF and CD31 causes angiogenesis for wound healing.

However, we need to further evaluate the expression of factors associated with wound healing at the protein or mRNA level to verify and expand our results. We also plan to further investigate how PDRN affects inflammation or remodeling during wound healing.

In conclusion, all of these results demonstrated the efficacy of PDRN as an intradermal injection for the promotion of wound healing. We expect that our PDRN intradermal injections will be helpful in the clinical study of delayed or chronic wound healing, because we demonstrated that PDRN injection led to increased expression of certain angiogenesis factors and supported the formation of collagen fibrils in mouse model.

\section{CONFLICTS OF INTEREST}

The authors have nothing to disclose.

\section{ORCID}

Tae-Rin Kwon, https://orcid.org/0000-0002-9892-7714

Sung Won Han, https://orcid.org/0000-0002-0040-3542

Jong Hwan Kim, https://orcid.org/0000-0001-6201-9602

Byung Chul Lee, https://orcid.org/0000-0002-6858-4853

Jae Min Kim, https://orcid.org/0000-0001-7333-3883

Ji Yeon Hong, https://orcid.org/0000-0002-5632-8449

Beom Joon Kim, https://orcid.org/0000-0003-2320-7621

\section{REFERENCES}

1. Oryan A, Alemzadeh E. Effects of insulin on wound healing: a review of animal and human evidences. Life Sci 2017; 174:59-67.

2. Galeano M, Bitto A, Altavilla D, Minutoli L, Polito F, Calò $M$, et al. Polydeoxyribonucleotide stimulates angiogenesis and wound healing in the genetically diabetic mouse. Wound Repair Regen 2008;16:208-217.

3. Jeong W, Yang CE, Roh TS, Kim JH, Lee JH, Lee WJ. Scar prevention and enhanced wound healing induced by polydeoxyribonucleotide in a rat incisional wound-healing model. Int J Mol Sci 2017; 18:E1698.

4. Barrientos S, Stojadinovic O, Golinko MS, Brem H, TomicCanic M. Growth factors and cytokines in wound healing. Wound Repair Regen 2008;16:585-601.

5. Ebrahimian TG, Pouzoulet F, Squiban C, Buard V, André M, Cousin B, et al. Cell therapy based on adipose tissue-derived stromal cells promotes physiological and pathological wound healing. Arterioscler Thromb Vasc Biol 2009;29:503-510. 
6. Smiell JM, Wieman TJ, Steed DL, Perry BH, Sampson AR, Schwab BH. Efficacy and safety of becaplermin (recombinant human platelet-derived growth factor-BB) in patients with nonhealing, lower extremity diabetic ulcers: a combined analysis of four randomized studies. Wound Repair Regen 1999;7:335-346.

7. Han G, Ceilley R. Chronic wound healing: a review of current management and treatments. Adv Ther 2017;34: 599-610.

8. Cianfarani F, Zambruno G, Brogelli L, Sera F, Lacal PM, Pesce $M$, et al. Placenta growth factor in diabetic wound healing: altered expression and therapeutic potential. Am J Pathol 2006;169:1167-1182.

9. Powell RJ, Goodney P, Mendelsohn FO, Moen EK, Annex BH; HGF-0205 Trial Investigators. Safety and efficacy of patient specific intramuscular injection of HGF plasmid gene therapy on limb perfusion and wound healing in patients with ischemic lower extremity ulceration: results of the HGF-0205 trial. J Vasc Surg 2010;52:1525-1530.

10. Altavilla D, Bitto A, Polito F, Marini H, Minutoli L, Di Stefano V, et al. Polydeoxyribonucleotide (PDRN): a safe approach to induce therapeutic angiogenesis in peripheral artery occlusive disease and in diabetic foot ulcers. Cardiovasc Hematol Agents Med Chem 2009;7:313-321.

11. Kim SK, Huh CK, Lee JH, Kim KW, Kim MY. H Histologic study of bone-forming capacity on polydeoxyribonucleotide combined with demineralized dentin matrix. Maxillofac Plast Reconstr Surg 2016;38:7.

12. Veronesi F, Dallari D, Sabbioni G, Carubbi C, Martini L, Fini M. Polydeoxyribonucleotides (PDRNs) from skin to musculoskeletal tissue regeneration via adenosine A2A receptor involvement. J Cell Physiol 2017;232:2299-2307.

13. Bitto A, Galeano M, Squadrito F, Minutoli L, Polito F, Dye $\mathrm{JF}$, et al. Polydeoxyribonucleotide improves angiogenesis and wound healing in experimental thermal injury. Crit Care Med 2008;36:1594-1602.

14. Squadrito F, Bitto A, Irrera N, Pizzino G, Pallio G, Minutoli $\mathrm{L}$, et al. Pharmacological activity and clinical use of PDRN. Front Pharmacol 2017;8:224.

15. Wu CH, Chang GY, Chang WC, Hsu CT, Chen RS. Wound healing effects of porcine placental extracts on rats with thermal injury. Br J Dermatol 2003;148:236-245.

16. Altavilla D, Squadrito F, Polito F, Irrera N, Calò M, Lo Cascio
$\mathrm{P}$, et al. Activation of adenosine $\mathrm{A} 2 \mathrm{~A}$ receptors restores the altered cell-cycle machinery during impaired wound healing in genetically diabetic mice. Surgery 2011;149:253-261.

17. Lee SJ, Lee HM, Ji ST, Lee SR, Mar W, Gho YS. 1,2,3,4,6Penta-O-galloyl-beta-D-glucose blocks endothelial cell growth and tube formation through inhibition of VEGF binding to VEGF receptor. Cancer Lett 2004;208:89-94.

18. Montesinos MC, Desai A, Chen JF, Yee H, Schwarzschild MA, Fink JS, et al. Adenosine promotes wound healing and mediates angiogenesis in response to tissue injury via occupancy of A(2A) receptors. Am J Pathol 2002;160:2009-2018.

19. Brem H, Stojadinovic O, Diegelmann RF, Entero $H$, Lee $B$, Pastar I, et al. Molecular markers in patients with chronic wounds to guide surgical debridement. Mol Med 2007;13: 30-39.

20. Brem H, Tomic-Canic M. Cellular and molecular basis of wound healing in diabetes. J Clin Invest 2007;117:12191222.

21. Reiber GE, Vileikyte L, Boyko EJ, del Aguila M, Smith DG, Lavery LA, et al. Causal pathways for incident lower-extremity ulcers in patients with diabetes from two settings. Diabetes Care 1999;22:157-162.

22. Christman AL, Selvin E, Margolis DJ, Lazarus GS, Garza LA. Hemoglobin A1c predicts healing rate in diabetic wounds. J Invest Dermatol 2011;131:2121-2127.

23. Emmerson E, Campbell L, Davies FC, Ross NL, Ashcroft GS, Krust A, et al. Insulin-like growth factor-1 promotes wound healing in estrogen-deprived mice: new insights into cutaneous IGF-1R/ERa cross talk. J Invest Dermatol 2012;132: 2838-2848.

24. Lima MH, Caricilli AM, de Abreu LL, Araújo EP, Pelegrinelli $\mathrm{FF}$, Thirone $\mathrm{AC}$, et al. Topical insulin accelerates wound healing in diabetes by enhancing the AKT and ERK pathways: a double-blind placebo-controlled clinical trial. PLoS One 2012;7:e36974.

25. Polito F, Bitto A, Galeano $M$, Irrera N, Marini $H$, Calò $M$, et al. Polydeoxyribonucleotide restores blood flow in an experimental model of ischemic skin flaps. J Vasc Surg 2012;55: 479-488.

26. Yan Y, Zhu L, Hong L, Deng J, Song Y, Chen X. The impact of ranibizumab on the level of intercellular adhesion molecule type 1 in the vitreous of eyes with proliferative diabetic retinopathy. Acta Ophthalmol 2016;94:358-364. 